\title{
REVIEWS
}

\section{Cancer immunotherapy: a paradigm shift for prostate cancer treatment}

\author{
Dev Karan, Jeffrey M. Holzbeierlein, Peter Van Veldhuizen and J. Brantley Thrasher
}

\begin{abstract}
Prostate cancer remains a significant health problem for men in the Western world. Although treatment modalities are available, these do not confer long-term benefit and are accompanied by deleterious side effects. Immunotherapy represents a valuable alternative to conventional treatments by inducing tumourspecific immune responses that control the growth of cancer cells. Sipuleucel-T is approved by the FDA as an immunotherapeutic agent for the treatment of patients with asymptomatic or minimally symptomatic castration-resistant prostate cancer (CRPC). Although this approval has raised cost-versus-benefit issues, it has provided proof of concept for the therapeutic potential of active immunotherapy approaches for metastatic CRPC. Numerous clinical studies have demonstrated clinical benefit using immunotherapy compared to traditional chemotherapy and several active immunotherapy approaches (at various developmental stages) have demonstrated the potential to change the face of prostate cancer treatment.
\end{abstract}

Karan, D. et al. Nat. Rev. Urol. 9, 376-385 (2012); published online 29 May 2012; doi:10.1038/nrurol.2012.106

\section{Introduction}

Among men, prostate cancer is the most common noncutaneous cancer and the second leading cause of cancer deaths in the Western world. ${ }^{1}$ Although this disease predominantly affects older men, a diagnosis of clinical-grade prostate cancer is not uncommon in men in their forties, or even in their thirties. ${ }^{2}$ Ethnicity is an independent risk factor for prostate cancer and African American men are at a 2.5-fold increased risk compared to white men. Thus, a variety of risk factors-including age, race, lifestyle, environment, and genetic predisposition-collectively contribute towards the development of prostate cancer. ${ }^{3}$ Given the complexity of its aetiology, it is important that the disease is detected in a localized state so that it can be targeted more effectively by curative therapies. Encouragingly, more than $90 \%$ of prostate tumours are detected prior to metastasis and patients are thus eligible for standard treatments such as radical prostatectomy, radiation therapy, cryotherapy, and active surveillance (watchful waiting). However, approximately $30 \%$ of these men will experience treatment failure and, ultimately, develop metastatic prostate cancer. Treatment for metastatic prostate cancer relies heavily on androgen ablation, which limits the growth of androgen-dependent cancer cells and results in clinical tumour control. Despite excellent initial responses to hormonal castration, prostate cancer cells eventually become castration resistant and the tumour continues to grow.

Novel treatment approaches for patients with progressive castration-resistant prostate cancer (CRPC) have been developed that involve chemotherapy with docetaxel followed by cabazitaxel. ${ }^{4}$ The CYP17 inhibitor

Competing interests

The authors declare no competing interests. abiraterone has been approved as a second-line therapy following failure of docetaxel-based chemotherapy. ${ }^{5}$ Unfortunately, the overwhelming majority of tumours still progress, leading to an estimated 30,000 deaths every year in the USA. ${ }^{1}$ This continues to pose a significant health threat to men in developed countries. In light of the increasing average life expectancy of men in the Western world, there is potential for an epidemic of advanced prostate cancer within the next 50 years, which could constitute a significant economical burden. ${ }^{6,7}$ Thus, novel treatments for advanced prostate cancer are desperately sought.

An increased understanding of basic immunology, combined with the identification of various tumourassociated antigens (TAAs), has fuelled the development of novel immunotherapy approaches for various cancers, including prostate cancer. In this Review, we describe several active immunotherapy approaches that have the potential to transform the way in which patients with prostate cancer are treated. We also highlight future areas of research that might help to improve the efficacy of immunotherapy for prostate cancer.

\section{Cancer immunotherapy}

The aim of immunotherapy is to induce immunity to diseases such as cancer using the body's own immune system. This approach is based on the concept of immune surveillance. ${ }^{8,9}$ According to this theory, the immune system constantly patrols the body for signs of abnormal (potentially precancerous) cell growth, and eliminates these abnormal cells before they become harmful to the host. During immune patrolling, various types of immune cells migrate towards the site of infection or injury, triggering an inflammatory response. In 
support of this hypothesis, chronic inflammation has been linked to the development of neoplastic formation in several organs and is widely considered to be a risk factor for cancer. ${ }^{10,11}$

Inflammation in the prostate is primarily regulated by T cells, B cells, and macrophages. ${ }^{12-14}$ Precancerous cells could harness the functions of infiltrating immune cells (released during the inflammatory response) to support cell growth. The escape of precancerous cells from immune surveillance could arise as a result of multiple factors, including weak immunogenicity, lack of proper communication between immune cells and precancerous cells, reduced (or absent) expression of major histocompatibility complex molecules, immunologic tolerance, increased levels of regulatory (suppressor) $\mathrm{T}$ cells, and suppressive activities produced by the neoplastic cells. Prolonged immune surveillance evasion could ultimately lead to the development of cancer. ${ }^{15-18}$

This neoplastic process is thought to occur in three distinct phases: elimination, equilibrium, and escape. ${ }^{19,20}$ Following clearance attempts by the immune system, abnormal (precancerous) cells that have survived the elimination phase enter a dormant phase (equilibrium), producing new cell variants but remaining clinically undetectable. With time, the new variants of abnormal cells-which are not generally susceptible to attack by immune cells-develop into clinically detectable tumours.

The ultimate goal of cancer immunotherapy is to identify and destroy tumour cells without exerting detrimental effects on the host. As cancer immunotherapy establishes a tumour-specific immunological memory, one focus of research has been to generate high-potency cytotoxic T lymphocytes (CTLs). Various immunotherapy approaches utilize whole cancer cells, parts of cancer cells (for example, RNA or DNA), or a particular TAA to stimulate the immune response. ${ }^{21-25}$ In particular, progress in this field has been driven by the identification of TAAs such as melanoma antigen family A (MAGEA) 1, MAGEA2, MAGEA3, melanoma antigen recognized by T cells 1 (MART1), glycoprotein 100 (gp100), carcinoembryonic antigen (CEA), human epidermal growth factor receptor 2 (HER2), mucin 1 cell surface associated antigen (MUC1), and prostate-cancer-specific antigens-including PSA, prostate stem cell antigen (PSCA), prostatic acid phosphatase (PAP), and prostatespecific membrane antigen (PSMA). Immune-based therapies that target these antigens are under investigation in various clinical trials to assess overall survival benefits. ${ }^{26-29}$

The number of tumour-antigen-specific CTLs has been shown to correlate with clinical response in various cancer vaccine studies to date. ${ }^{30,31}$ Although there are no defined criteria for measuring the CTL response that is mounted following immunization, increased expression of interferon (IFN) $-\gamma$-which is produced by antigenic CD8 T cells - and increased in vitro CTL activity are routinely used to define the potential of the developed antitumour immunity. Over time, we have learned that the utilization of effector CTLs to provide potent antitumour

\section{Key points}

- Prostate cancer is responsive to active immunotherapy, as evidenced by the clinical efficacy of sipuleucel-T and PROSTVAC ${ }^{\circledR}$-VF vaccination

- Sipuleucel-T is the first FDA-approved immunotherapy option for the treatment of minimally symptomatic or asymptomatic metastatic castration-resistant prostate cancer

- Cancer vaccines containing adenovirus and plasmid DNA are emerging as potential approaches for active immunotherapy; these are at various stages of development and clinical evaluation

- Overall survival remains the gold-standard study end point; alternative end points (such as novel biomarkers) are sought for patients with early-stage disease

- Immunotherapeutic approaches might be most effective for patients with minimal residual disease and for use early in the treatment paradigm

- Future studies should focus on identifying which patients are most likely to benefit from treatment, as well as on the development of combination therapies and novel multitargeted approaches that can increase survival advantage

immunity depends on multiple factors, including antigen selection and delivery method.

\section{Antibody-based targeted immunotherapy}

Therapies targeting CTL-associated antigen 4 (CTLA4) have been a particular research focus for the treatment of various solid tumours, including prostate cancer. ${ }^{32-35}$ CTLA4 is a cell-surface molecule on T cells that is engaged in the suppression of T-cell activation. Circulating T cells demonstrate increased expression of CTLA4 in patients with cancer; ${ }^{36}$ thus, targeted inhibition of CTLA 4 might induce the function of effector $\mathrm{T}$ cells. Ipilimumab and tremelimumab - two antibodies that target and inhibit CTLA4-were initially tested in patients with melanoma. In phase III clinical trials of patients with advanced melanoma, only ipilimumab improved overall survival compared to standard chemotherapy (no survival benefit was evident with tremelimumab). ${ }^{37,38}$ On this basis, Small et al. ${ }^{35,39}$ evaluated the effect of administering ipilimumab to patients with CRPC, both as monotherapy and in combination with granulocyte-macrophage colonystimulating factor. When given as monotherapy, a single dose of $3.0 \mathrm{mg} / \mathrm{kg}$ was safe and tolerable. However, the toxicity of combination therapy was a limiting factor, despite its clinical efficacy.

In another trial, a combination of ipilimumab and the gp100 peptide vaccine Gp100:209-217(210M) was associated with improved overall survival in patients with metastatic melanoma compared to monotherapy with either agent. ${ }^{38}$ This suggests that combining antibody-based therapy with selected antigen targets might improve the therapeutic potential of immunotherapy for other solid tumours. Two phase I studies have demonstrated the safety of administering ipilimumab combined with either PROSTVAC ${ }^{\circledR}$-VF or GVAX $^{\circledR}$ prostate cancer vaccines to patients with metastatic CRPC. ${ }^{40,41}$ It is likely that a phase II trial will follow to establish the efficacy of these combinations. Currently, two phase III clinical trials are investigating the efficacy of ipilimumab therapy for metastatic CRPC; either alone ${ }^{42}$ or in combination with radiotherapy. ${ }^{43}$

Another promising candidate for antibody-based targeted therapy is denosumab-a humanized monoclonal 
antibody against the receptor activator of $\mathrm{NF \kappa B}$ ligand (RANKL) that is used for the prevention of skeletalrelated events in patients with bone metastases. In a phase III double-blind randomized study, denosumab treatment was associated with a reduction in skeletal fractures compared to zoledronic acid therapy in men with CRPC and associated bone metastases. ${ }^{44}$

\section{Dendritic-cell-based vaccination}

A new generation of cancer immunotherapies has been developed based on our increased understanding of dendritic cells, which play a central role in the development of effector $\mathrm{T}$ cells. Active immunization methods that induce TAA-specific CTLs using ex vivo dendritic cells loaded with antigen, cell-based, plasmid DNA, or viral vaccines have undergone clinical evaluation for the treatment of various cancers, including prostate cancer. The question regarding whether dendritic cells are a homogeneous cell population with multiple functions or a heterogenous group of cells that exhibit differences in morphology, function, and localization, remains unanswered. Despite increasing evidence to show that dendritic cells display varying degrees of plasticity and cannot be rigidly categorized according to a particular phenotype ${ }^{45}$ these cells are most commonly classified as either plasmacytoid or myeloid, based on phenotypic markers. ${ }^{46}$

Dendritic cells are professional antigen-presenting cells (APCs) that process and present TAAs to T cells, stimulating the production of a costimulatory signal and triggering the development of effector (tumour-reactive) $\mathrm{T}$ cells. Various studies have examined the direct activation and antigen loading of dendritic cells in ex vivo systems and demonstrated an induction of antigenspecific immune response. ${ }^{47}$ In one study, autologous dendritic cells from patients with CRPC were loaded with antigenic peptides derived from four different PSAs (PSCA, PAP, PSMA, and PSA). These antigen-loaded dendritic cells were injected intradermally in sets of six immunizations (three injections into each thigh) every other week for 3 months, followed by monthly booster injections where appropriate (total treatment duration of up to 20 weeks). This vaccination regimen was well tolerated and induced significant CTL responses against all PSAs. Furthermore, it was associated with a prolongation in PSA doubling time. ${ }^{48}$

Similarly, a phase I clinical trial studied the effects of administering dendritic cells transfected with PSA mRNA to patients with metastatic CRPC. ${ }^{49}$ For this treatment approach, autologous dendritic cells were generated from peripheral blood mononuclear cells (PBMCs). ${ }^{50}$ After 7 days in culture, the dendritic cells were co-incubated with a vector containing PSA mRNA (pGEM4/PSA/A64) to enable transient transfection. These transfected cells were washed and incubated overnight to allow time for the expression and presentation of the PSA antigen. Patients with metastatic CRPC were vaccinated with PSA-mRNA-transfected dendritic cells at biweekly intervals to assess the safety and toxicity of this approach. The study revealed a small, but statistically significant, decrease in PSA velocity in treated patients.

\section{Sipuleucel-T}

Sipuleucel-T is the only immunotherapy approved by the FDA for the treatment of asymptomatic or minimally symptomatic metastatic CRPC. It is an autologous cellular immunotherapy that has been designed to stimulate a patient's own immune response against prostate cancer. It acts in a similar way to a vaccine and is thus referred to as a dendritic-cell-based vaccine immunotherapy. Treatment begins with leukapheresis to isolate a patient's PBMCs, which are a mixture of APCs, T cells, B cells, and other immune cells. These PBMCs are then activated ex vivo by the recombinant human PAP/GM-CSF fusion protein (PA2024) and reinfused back into the patient to target and treat the prostate cancer. ${ }^{51}$ Each dose of sipuleucel-T contains a minimum of 40 million cells expressing the costimulatory molecule CD54. The number and cellular composition of the final dose depends on the composition of the cells harvested during leukapheresis. ${ }^{52}$ Cells expressing CD54 are considered to be the 'active ingredient' of the treatment as CD54 is predominantly localized to APCs, which are responsible for antigen uptake..$^{53}$ Although the precise mechanism of action is unknown, it is thought that PAP/GM-CSF is internalized by the APCs during ex vivo culture and PAP-specific peptides-such as major histocompatibility complex (MHC) class I and class II peptides-are expressed on the surface of the APCs.

In phase III studies, treatment with sipuleucel-T resulted in a median survival advantage of 4.1 months, with a 3-year survival rate of $31.7 \%$ (compared with $23.0 \%$ for patients receiving placebo). ${ }^{51}$ However, no difference was observed between the two study arms in terms of progression-free survival (PFS), time to clinical progression, or time to PSA progression. The reason for the dissociation between overall survival and measurable antitumour effect is unclear. It is, therefore, difficult to determine which patients are likely to benefit from treatment and all patients are treated empirically at present.

The exclusivity of FDA approval for sipuleucel-T immunotherapy for prostate cancer has raised issues regarding cost versus benefit. Although traditional chemotherapy is expensive, treatment cost can be regulated by the discontinuation of therapy when this proves to be ineffective. Sipuleucel-T, on the other hand, is administered in full at the start of treatment, prior to any assessment of benefit. Moreover, evidence is lacking to support a direct correlation between the proposed mechanism of action of sipuleucel-T (via induction of antigenic immune and tumour responses) and the observed clinical benefit of this drug.

To better understand the functional activity of sipuleucel-T, a phase II study is evaluating the magnitude of immune response to different concentrations of PA2024 antigen in patients with metastatic CRPC. ${ }^{54}$ Another phase II study, set up to measure antigen-specific immune responses over time, is currently monitoring men who have failed primary therapy and are given sipuleucel-T before or after androgen deprivation therapy. ${ }^{55}$ In the wake of encouraging preliminary results regarding novel combination therapy with antiandrogens, a trial 
Table 1 | Results of selected immunotherapy trials for patients with prostate cancer

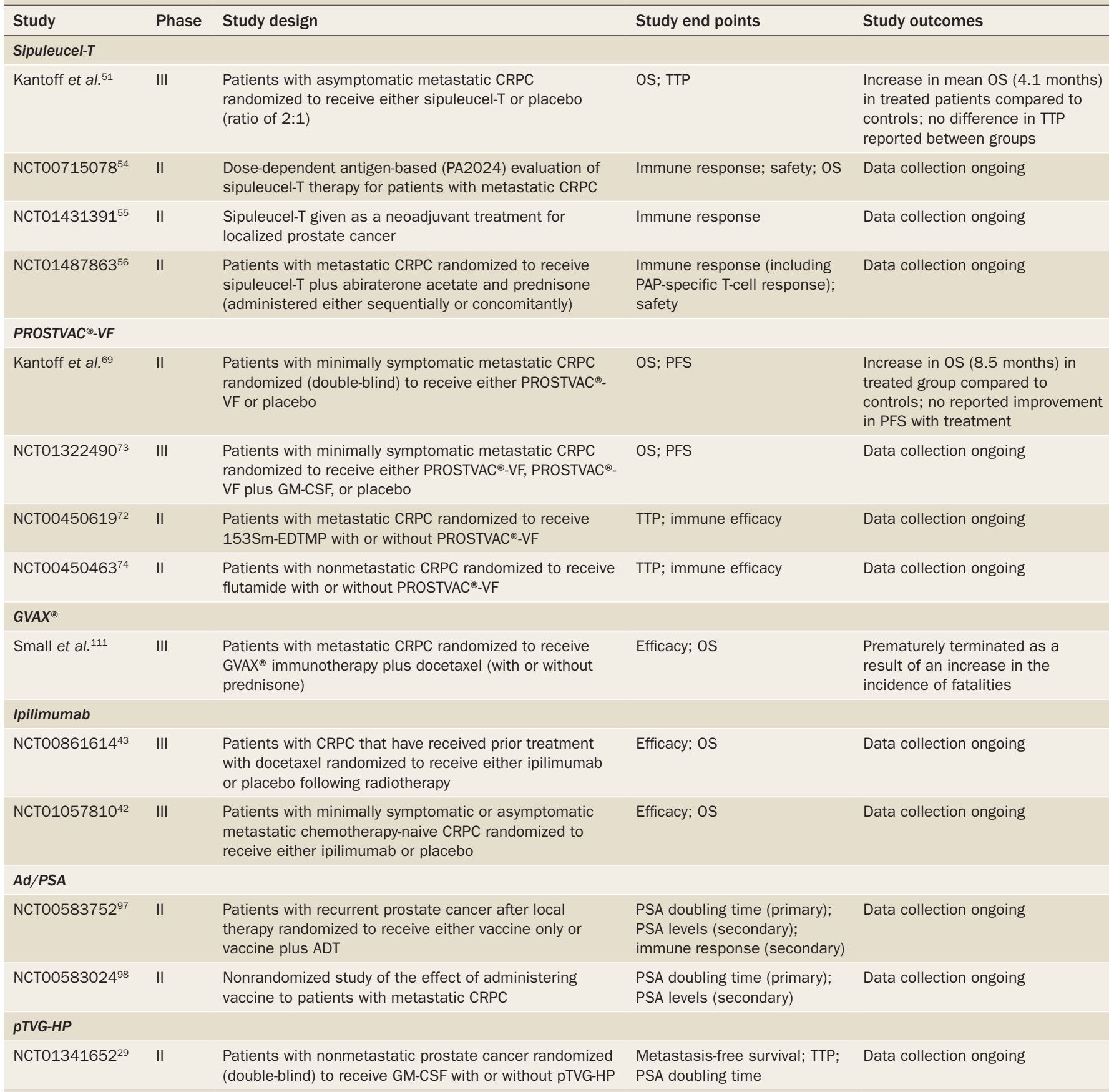

Abbreviations: Ad/PSA, replication-deficient PSA-expressing adenovirus vaccine; CRPC, castration-resistant prostate cancer; GM-CSF, granulocyte-macrophage colony-stimulating factor; GVAX ${ }$, GM-CSF modified tumour cell vaccine; OS, overall survival; PAP, prostatic acid phosphatase; PFS, progression-free survival; pTVG-HP, plasmid DNA containing PAP antigen; TTP, time to progression.

has been instigated to assess the safety and efficacy of administering sipuleucel-T-given either simultaneously or sequentially with antihormonal agents such as abiraterone acetate plus prednisone-to patients with metastatic CRPC (Table 1). ${ }^{56}$

\section{Microbial vectors for cancer immunotherapy}

The ability of microbial vectors to carry foreign antigens has been harnessed by various vaccine approaches to targeted immunotherapy. Bacterial strains have been engineered to express cancer-specific antigens and cytokines, as well as prodrug-converting cytokines. A variety of microbial vectors-including BCG, Listeria monocytogenes (LM), Salmonella, Escherichia coli, and Shigella-have demonstrated efficacy as vaccine vectors in preclinical animal models of infectious disease and cancer. ${ }^{57}$ These microbial vectors are useful because of their strong ability to infect APCs and induce potent antitumour responses to targeted antigens. However, utilization of microbial agents as delivery vehicles is limited because the properties of these vaccine vectors have not yet been fully characterized. 


\section{Listeria monocytogenes}

Although the clinical development of bacteria-based vectors has generally lagged behind that of other currently used vaccine approaches, several unique characteristics of Listeria monocytogenes have focused attention on the use of this vector for active immunotherapy, including its ability to induce both arms of the immune response (innate and adaptive). ${ }^{58}$ As L. monocytogenes can access both the phagosomal and cytosolic compartments of APCs, L. monocytogenesdelivered antigens can be presented in the context of MHC class I and class II molecules, resulting in stronger immune responses. ${ }^{59,60} \mathrm{~A}$ number of live attenuated strains of L. monocytogenes have been developed to express several human antigens, including HER2, human papilloma virus (HPV) E7 antigen, MAGE, and PSA ${ }^{61-64}$ Preclinical studies of PSA-expressing $L$. monocytogenes have shown that this strain is immunogenic and could have therapeutic potential for treating prostate cancer. Interestingly, the PSA-expressing L. monocytogenes strain was more effective at inducing tumour regression than vaccinia virus vectors carrying human PSA. ${ }^{65,66}$ Although this strain is in the early stages of development, trials of patients with metastatic cervical cancer who have been treated with $L$. monocytogenes expressing the HPV E7 antigen and patients with lung metastases treated with human mesothelin (CRS-207) have indicated the safety and tolerability of L. monocytogenes-based vaccination. ${ }^{67,68}$ Although additional clinical trials are needed to confirm these initial promising results, it seems that $L$. monocytogenes-based vaccination could induce immune activation with a potential clinical benefit against prostate cancer.

\section{PROSTVAC ${ }^{\circledR}$-VF}

Another novel form of immunotherapy for prostate cancer that utilizes a microbial vector is PROSTVAC $^{\circledR}$-VF. This vaccine approach uses a 'primeboost strategy', involving vaccinia and fowlpox viral vectors that express a PSA-targeted molecule and a triad of costimulatory molecules (B7.1, ICAM-1, and LFA-3) - termed the vaccinia-PSA-TRICOM and fowlpox-PSA-TRICOM vaccines. ${ }^{69,70}$ Using this approach, the patient's immune system is first primed with vacciniaPSA-TRICOM. Subsequent vaccination with fowlpox-PSA-TRICOM boosts the CTL immune response against PSA-expressing tumour cells. The efficacy of PROSTVAC $^{\circledast}$-VF has been analyzed in a randomized multicenter, double-blind phase II study of patients with metastatic CRPC ( $n=125)$. Although no improvement in PFS - the primary end point of the study-was observed in the treated cohort, patients receiving PROSTVAC ${ }^{\circledR}$-VF therapy experienced an 8.5 month improvement in overall survival relative to controls. ${ }^{69}$

In a separate randomized phase II study of 64 patients treated with PROSTVAC ${ }^{\circledast}$-VF, $45.3 \%$ of patients remained free of PSA progression at 19 months (primary end point of the study) and $78.1 \%$ of patients demonstrated a clinical benefit in PFS compared to controls. ${ }^{70}$ Analysis of PSA-specific immune response revealed an increased number of PSA-specific T cells relative to the baseline (PBMCs collected prior to immunization) in $46 \%$ of treated patients. However, no differences were observed between the treated and untreated cohorts in relation to anti-PSA antibody response..$^{70} \mathrm{In}$ a similar, albeit smaller, study of 32 patients, vaccination with PROSTVAC ${ }^{\circledR}$-VF was linked to an increase in levels of PSA-specific T cells in association with improved survival. ${ }^{71}$ Encouraged by the outcomes of these trials, as well as demonstrations of PSA-specific immune responses, PROSTVAC ${ }^{\oplus}$-VF is currently being tested in a phase III study, as well as two phase II combination therapy studies (Table 1)..$^{72-74}$

\section{Adenoviruses}

Increasing attention is being directed towards the use of adenoviruses as expression vectors and recombinant virus vaccines. For gene-therapy-based and vaccinebased approaches, adenovirus serotype 5 (Ad5) has been the most extensively studied vector. ${ }^{75-77}$ The Ad5-vector has been genetically modified for vaccine therapy to allow insertion of foreign genes. Current vectors have deletions in the E1a and E1b regions, as well as a partial deletion of the E3 region. Ad5-vector-based vaccines for prostate cancer are produced by inserting the gene for full-length human PSA into a replication-deficient Ad5-vector to create Ad5-PSA, which induces a strong anti-PSA CD8 ${ }^{+}$T-cell response. ${ }^{78,79}$ Moreover, Ad5-PSA has been shown to induce stronger anti-PSA immune responses than other viral PSA vaccines, including vaccinia virus vaccines (both replication competent and replication deficient) and a canarypox vaccine. ${ }^{79,80}$ The Ad5-vector is able to transduce DNA between a wide range of dividing and nondividing cells types, making this gene delivery system a valuable tool for vaccine therapy ${ }^{81,82}$ Furthermore, it is genetically and biochemically well characterized and can be produced at high titres under good manufacturing practice conditions. ${ }^{83,84}$

Adenovirus-based cancer vaccines have been used to induce active immunization in murine models for various types of cancer, as well as in some clinical studies. ${ }^{85-88} \mathrm{Ad} 5$-vectors that express prostate cancer antigens such as PSA, PSMA, PSCA, or six-transmembrane epithelial antigen of the prostate (STEAP) have all demonstrated therapeutic effects in preclinical models. ${ }^{89}$ A study with 10-year follow-up safety data has supported the continued use and development of adenoviruses as gene delivery vehicles. ${ }^{90,91}$ The Vaccine Research Center at the US National Institutes of Health has developed several candidates for cancer vaccines based on the Ad5-vector and has performed biodistribution and toxicological safety studies that support the investigational use of Ad5-based vaccine candidates in humans. ${ }^{92}$ In addition, wild-type adenovirus infections are extremely common in the general population, providing a welldocumented safety record. Indeed, it could be argued that adenovirus-mediated gene delivery is associated with lower morbidity than most currently used treatments for prostate cancer including surgery, hormonal therapy, and chemotherapy. 
Overcoming anti-adenovirus immunity

Despite potential advantages, the use of the Ad5-vector as a delivery vehicle is hampered by the prevalence of preexisting anti-adenovirus immunity in human populations. ${ }^{93,94}$ To improve the immunogenicity of the Ad5-vector, the adenovirus can be administered with GELFOAM ${ }^{\circledR}$ to enhance CTL activity in the presence of anti-Ad5 immunity. ${ }^{95}$ This finding is important as most humans have pre-existing levels of anti-adenovirus antibodies, resulting from prior natural exposure to the virus.

In support of Ad5-vector-based vaccination, a phase I trial showed the development of an anti-PSA T-cell response in $68 \%$ of patients with prostate cancer treated with the Ad5-PSA vaccine injected with GELFOAM ${ }^{\circledR}{ }^{\circledR 6}$ This study, which included 32 patients with advanced CRPC, demonstrated the safety of Ad5-vector vaccination for prostate cancer. Following on from these results, a phase II continuation of this study is currently evaluating the efficacy of Ad5-PSA treatment for patients with a rising PSA after local therapy, and for patients with CRPC. ${ }^{97,98}$ Interim results for 44 patients-presented at the American Association for Cancer Research annual meeting in 2012 - showed that three injections of Ad5-PSA vaccine in GELFOAM $^{\circledR}$ (administered at intervals of 30 days) induced anti-PSA T-cell responses above the baseline (pre-immunization) level in $100 \%$ of patients with a rising PSA after local therapy and $67 \%$ of patients with CRPC. The majority (64\%) of patients demonstrated an increase in PSA doubling time. ${ }^{99}$ These observations suggest that the use of GELFOAM ${ }^{\circledR}$ with Ad5-based vaccines induces an immune $\mathrm{T}$-cell response against the self-antigen and circumvents pre-existing anti-Ad5 immunity.

\section{In vivo dendritic cell targeting using Ad5-vectors}

It is widely accepted that dendritic cells have a pivotal role in the development of effector T cells; thus, the development of immune-based therapies has focused on the activation of dendritic cells. Despite advantages of autologous dendritic-cell-based vaccination, the status of dendritic cell maturation during the preparation of a dendritic-cell-based vaccine is a critical issue that presents many challenges. ${ }^{100,101}$ Vaccine development is constrained by clinical practicalities as the vaccine must be patient-specific, and vaccine preparation techniques are cumbersome, laborious, and costly. Furthermore, in vivo dendritic cell migration is a complex and tightly regulated process that involves multiple cellular components, including cytokines, chemokines, and adhesion molecules, and ex vivo cultured and modified (antigenloaded) dendritic cells have demonstrated poor migration in vivo, adversely affecting the efficacy of the vaccine. ${ }^{102}$

Various studies have shown that the Ad5-vector is capable of modulating dendritic cell maturation by increasing expression of MHC antigens and costimulatory molecules, making this delivery system a valuable tool for studying disease pathology, vaccine therapy mechanisms, and potential clinical use. ${ }^{81,82}$ It is likely that adenoviruses, which contain adjuvant-like properties, provide the costimulatory signals necessary to generate a strong antigen-specific effector T-cell response.
Interaction of CD40 with its ligand (CD40L) constitutes one of the basic costimulatory signals that activate dendritic cells to generate CTLs with enhanced antitumour activity. Multiple studies have utilized adenoviruses carrying CD40L to activate CD40 on dendritic cells, generating high-potency tumour-reactive $\mathrm{CD} 8^{+} \mathrm{T}$ cells. ${ }^{103,104}$ Therefore, Ad5-vector-based in vivo targeting of tumourspecific antigens, combined with dendritic cell activation, might represent an important immunotherapy approach.

The use of adenoviruses as delivery vehicles potentially infects dendritic cells in vivo to induce an antigen-specific effector T-cell response. In mouse models, subcutaneous injection of bone-marrow-derived dendritic cells (BMDCs) treated with Ad5-vector carrying a model antigen induced immune and antitumour responses. ${ }^{105,106}$ Bone marrow cells were harvested by flushing the murine femurs and tibias to generate dendritic cells. Following overnight treatment with Ad5-OVA (Ad5-vector carrying ovalbumin as a model antigen), BMDCs were washed with phosphate buffer saline and injected subcutaneously into treatment-naive mice. A week after injection, immune response (level of IFN- $\gamma$-secreting $\mathrm{CD}^{+} \mathrm{T}$ cells) was measured by in vitro assay for BMDC-immunized mice. Mice challenged with OVA-expressing EG7 tumour cells demonstrated significant inhibition of tumour growth. ${ }^{106}$ These observations further suggest that the Ad5-vector can be used as a vaccine system to deliver cancer-specific antigens by infecting ex vivo dendritic cells, which then process and present antigens to induce a protective immune response in vivo upon reinsertion.

\section{Multigene-targeted immunotherapy}

The generation of a tumour-specific $\mathrm{CD} 8^{+} \mathrm{T}$-cell response is generally considered to be the most potent immunotherapy approach. It is well documented that adenoviruses can be used as vehicles for antigen delivery, as well as vaccine carriers, to strongly induce cytotoxic T-cell responses, which are actively involved in the destruction of tumour cells that express cognate antigens. However, vaccines for prostate cancer have only, as yet, employed a single antigen, despite the fact that patients have often been heavily pretreated and frequently harbour advanced tumour burdens with a high propensity for changing their expressed antigens. Prostate cancer is a heterogeneous multifocal disease that undergoes significant genetic changes during its transition from a hormone-responsive to a hormone-refractory malignancy. Thus, the use of a single-antigen-based immunotherapy might not provide an efficient immune response against the prostate tumour cells directing the immunoediting process.

To expand the range of antitumour responses, some investigators have loaded dendritic cells with multiple peptides ex vivo to generate antitumour immunity. ${ }^{48,107}$ However, multipeptide-loaded dendritic cells lack the appropriate costimulatory molecules required for activating effector $\mathrm{T}$ cells, which limits the antitumour response. ${ }^{108}$ Using a preclinical mouse model, a recent study demonstrated the therapeutic benefit of administering dendritic cells loaded with a virus-expressed 


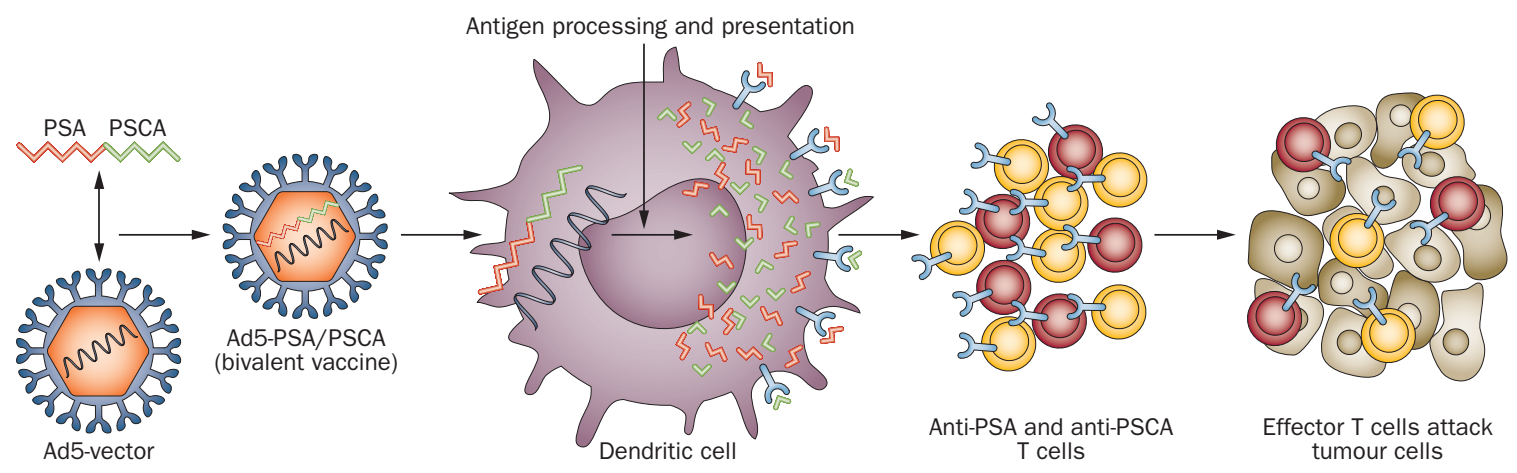

Figure 1 | Schematic presentation of the mechanism of action of an adenovirus vector encoding the PSA-PSCA fusion protein. Antitumour immunity develops via simultaneous targeting of multiple tumour-associated antigens.

cDNA library specific to prostate cancer. ${ }^{109}$ Clinical studies are now needed to confirm the safety and efficacy of this approach in humans.

\section{GVAX ${ }^{\circledR}$}

GVAX $^{\circledR}$ is a GM-CSF modified tumour cell vaccine produced by engineering the prostate tumour cell lines PC3 and $\mathrm{LNCaP}$ to secrete GM-CSF. ${ }^{28}$ This vaccine, which is one of the most extensively studied vaccines for treating prostate cancer, was designed to induce a broad range of prostate tumour-specific immune responses. Despite promising early results, ${ }^{110}$ Vital-2-a GVAX ${ }^{\circledR}$ phase III trial-was terminated because of safety concerns. ${ }^{111}$ The reason for this is not entirely clear. As GVAX $^{\circledR}$ vaccination utilizes irradiated prostate tumour cells (cellular vaccine), the immune responses associated with this approach could be undesirable and might outweigh the clinical benefit. It is highly likely that uncontrolled levels of GM-CSF in both GVAX ${ }^{\circledast}$ and sipuleucel-T could cause counter-regulatory immune responses, as higher levels of GM-CSF are also associated with an increased number of suppressor T cells. ${ }^{112,113}$ Administering GVAX $^{\circledR}$ vaccination concurrently with ipilimumab therapy has demonstrated safety in a phase I trial of patients with metastatic CRPC. However, development of autoimmune response remains a concern. ${ }^{41}$

\section{Ad5-PSA/PSCA bivalent vaccine}

As the heterogeneity of prostate cancer might limit the therapeutic benefit of single-antigen-based targeted therapies, one alternative approach is to amplify the potential of antitumour immunity using a combination of PSA and PSCA antigens, which are both highly specific to the prostate. PSCA is stably expressed in advanced prostate cancer and has been proposed as a prospective therapeutic target for CRPC. ${ }^{114-117}$ It is likely that initiating a strong line of attack on a heterogeneous tumour mass by inducing an amplified immunity against multiple target antigens will significantly reduce the ability of tumour cells to escape immune surveillance.

An Ad5-based bivalent vaccine encoding a fusion of human PSA and PSCA cDNA (Ad5-PSA/PSCA) has been proposed as a potential therapy for prostate cancer. ${ }^{106}$ The Ad5-PSA/PSCA vaccine infects dendritic cells and the cytomegalovirus promoter of the adenovirus drives the synthesis of a PSA-PSCA fusion protein. This protein undergoes antigen processing, leading to the presentation of PSA and PSCA gene-specific peptides on the surface of dendritic cells and the induction of an antigen-specific T-cell response (Figure 1). The Ad5-PSA/PSCA vaccine has been shown to simultaneously induce PSA-specific and PSCA-specific CD8 ${ }^{+}$ T-cell responses (determined by IFN- $\gamma$ cytokine secretion) in mice. Furthermore, this approach induced a strong antitumour immunity that inhibited the growth of established tumours coexpressing cognate antigens; $80 \%$ of mice were tumour free following treatment. ${ }^{106}$ These observations also suggest that multigene-targeted TAAspecific CTLs can be generated simultaneously (with an additive antitumour effect) to improve therapeutic efficacy of an immunotherapy approach. This preclinical study provides a strong rationale for further developing and investigating the Ad5-PSA/PSCA bivalent vaccine to determine safety, dose tolerance, and clinical proof of concept in patients with prostate cancer.

\section{Combining immunotherapy with other treatment}

Combining immunotherapy with an adjuvant chemotherapeutic drug, radiation therapy, or an immunosuppressant could potentially reduce tumour burden and improve overall survival. Use of an adjuvant therapy, such as the Toll-like receptor 9 (TLR9) agonist CpG, with Ad5-based immunotherapy (for example, Ad5-PSA/ PSCA) might enhance the potential of the antitumour response by inducing both innate and adaptive arms of the immune system. Immunotherapy approaches involving sipuleucel-T and PROSTVAC ${ }^{\circledR}$-VF in combination with chemotherapy drugs are also being evaluated. ${ }^{118,119}$

Given that inflammation could support the development of a tumour, simultaneous targeting of inflammation in combination with immunotherapy might improve the therapeutic efficacy of a vaccine. Prednisone is an anti-inflammatory agent used for prostate cancer treatment in combination with other chemotherapy drugs. However, this drug is known to weaken the immune system, so the risks of combination therapy might outweigh the beneficial effects. An ongoing study is analyzing the effect of administering prednisone in combination with sipuleucel-T on the development of immune response. ${ }^{56}$ 
Following immunotherapy with radiation therapy could be advantageous because the release of cell products from tumour cells killed during radiation therapy will further activate the immune system, which has already been triggered by immunotherapy. Similarly, depletion of immune suppressor cells will reduce the suppressive activity of a faulty immune system and provide more space for the expansion of effector $\mathrm{T}$ cells, induced following immunotherapy.

Overall, immunotherapy is a promising approach that could significantly reduce the mortality rate associated with CRPC when used in combination with other treatment modalities. However, such combinations need to be tested step-by-step in preclinical and clinical settings. Study design is critical when evaluating the clinical benefit of such combinations as the use of chemotherapy drugs could have a negative impact on the development of immune responses.

\section{Conclusions}

Immunotherapy has substantial potential as a nontoxic method of specifically and systematically destroying tumour cells. Multiple approaches for immunotherapy are emerging, including the use of plasmid DNA, microbial vectors, and direct DC manipulations. The success of current cancer immunotherapies-including adoptive cell transfer and cytokine therapy for melanoma, cytokine therapy for renal cell carcinoma, trastuzumab for breast cancer, sipuleucel-T for prostate cancer, and various antibody-based cancer therapies-supports the continuation of research in this field. ${ }^{120,121}$ Among these approaches, sipuleucel-T and PROSTVAC ${ }^{\circledR}$-VF have been the most successful for treating patients with prostate cancer, with a proven record of safety and clinical benefit.

Analyzing the efficacy of immunotherapy (or therapeutic combinations that include immunotherapy) remains a challenge. Currently, therapeutic responses are monitored based on changes in PSA or PSA-related parameters (including PSA velocity or doubling time), along with other clinical surrogates. Although well established as an indicator of disease progression, PSA-related changes are not always reliable for predicting the efficacy of therapeutic treatments. Currently, no validated immunologic biomarkers have been identified that can reliably predict clinical response following immunotherapies. Immunologic parameters for measuring the clinical success of immunotherapy are limited to antigen-specific $\mathrm{CD}^{+}{ }^{+} \mathrm{T}$-cell levels or IFN- $\gamma$ secretion. ${ }^{122,123}$ Analysis of PSA-specific or PAP-specific immune responses following PROSTVAC ${ }^{\circledR}$-VF or sipuleucel-T vaccines has established a limited correlation between these variables and reported clinical benefits. It has been suggested that continuous immune modulation by the initially induced antitumour response that follows vaccination leads to improved overall survival. ${ }^{124}$ This power struggle between the immune system and the tumour might keep the tumour burden in a state of equilibrium. An objective decrease in the tumour burden would then only occur if the immune system overpowered the tumour cells. Immunotherapeutic approaches might, therefore, be most effective against minimal residual disease and when used at an early stage of the treatment paradigm.

As immunotherapy works by inducing an immune system response against cancer cells, a longer follow-up duration could be required to observe clinical benefit and, therefore, overall survival remains the standard study end point. This lack of predictive biomarkers limits our ability to effectively manage the treatment of metastatic CRPC, especially when differentiating between patients who are responding to therapy and those who are not. Thus, the identification of immunological parameters or novel biomarkers that could be used to determine the efficacy of immunotherapy would have significant clinical utility and drive further advances in this field.

Review criteria
The literature for this Review was primarily searched
through PubMed using the terms "prostate cancer
immunotherapy", "adenovirus based immunotherapy",
"sipuleucel-T" and "Prostvac-VF". Relevant articles from
the last 10 years were identified. Cross references
were checked and relevant articles retrieved. In cases
when publications were not available through PubMed,
LISTA (EBSCO) and Web of Science databases were also
checked. Information about ongoing clinical trials of
active immunotherapy for prostate cancer was obtained
from http://clinicaltrials.gov.

1. Siegel, R., Naishadham, D. \& Jemal, A. Cancer statistics, 2012. CA Cancer J. Clin. 62, 10-29 (2012).

2. National Cancer Institute. Surveillance epidemiology and end results [online], http:// seer.cancer.gov/statfacts/html/prost.html (2012).

3. Karan, D., Thrasher, J. B. \& Lubaroff, D. Prostate cancer: genes, environment, immunity and the use of immunotherapy. Prostate Cancer Prostatic Dis. 11, 230-236 (2008).

4. Antonarakis, E. S. \& Eisenberger, M. A. Expanding treatment options for metastatic prostate cancer. N. Engl. J. Med. 364, 2055-2058 (2011).

5. Djavan, B. et al. Immunotherapy in the treatment of advanced prostate cancer. Can. J. Urol. 18, 5865-5874 (2011).
6. Roehrborn, C. G. \& Black, L. K. The economic burden of prostate cancer. BJU Int. 108, 806813 (2011).

7. Stokes, M. E., Ishak, J., Proskorovsky, I., Black, L. K. \& Huang, Y. Lifetime economic burden of prostate cancer. BMC Health Serv. Res. 11, 349 (2011).

8. Burnet, F. M. Concept of immunological surveillance. Progr. Exp. Tumor Res. 13, 1-27 (1970).

9. Burnet, M. Cancer: a biological approach. III. Viruses associated with neoplastic conditions. IV. Practical applications. Br. Med. J. 1, 841-847 (1957).

10. Coussens, L. M. \& Werb, Z. Inflammation and cancer. Nature 420, 860-867 (2002).

11. Nelson, W. G., De Marzo, A. M., DeWeese, T. L. \& Isaacs, W. B. The role of inflammation in the pathogenesis of prostate cancer. J. Urol. 172, S6-S12 (2004).

12. Robert, G. et al. Inflammation in benign prostatic hyperplasia: a 282 patients' immunohistochemical analysis. Prostate 69, 1774-1780 (2009).

13. Theyer, G. et al. Phenotypic characterization of infiltrating leukocytes in benign prostatic hyperplasia. Lab. Invest. 66, 96-107 (1992).

14. Sottnik, J. L., Zhang, J., Macoska, J. A. \& Keller, E. T. The PCa tumor microenvironment. Cancer Microenviron. 4, 283-297 (2011).

15. Ahmad, M., Rees, R. C. \& Ali, S. A. Escape from immunotherapy: possible mechanisms that influence tumor regression/progression. Cancer Immunol. Immunother. 53, 844-854 (2004).

16. Miller, A. M. \& Pisa, P. Tumor escape mechanisms in prostate cancer. Cancer Immunol. Immunother. 56, 81-87 (2007). 
17. Poggi, A. \& Zocchi, M. R. Mechanisms of tumor escape: role of tumor microenvironment in inducing apoptosis of cytolytic effector cells. Arch. Immunol. Ther. Exp. (Warsz) 54, 323-333 (2006).

18. Sadun, R. E. et al. Immune signatures of murine and human cancers reveal unique mechanisms of tumor escape and new targets for cancer immunotherapy. Clin. Cancer Res. 13, 4016-4025 (2007).

19. Schreiber, R. D., Old, L. J. \& Smyth, M. J. Cancer immunoediting: integrating immunity's roles in cancer suppression and promotion. Science $\mathbf{3 3 1}$, 1565-1570 (2011).

20. Dunn, G. P., Bruce, A. T., Ikeda, H., Old, L. J. \& Schreiber, R. D. Cancer immunoediting: from immunosurveillance to tumor escape. Nat. Immunol. 3, 991-998 (2002).

21. Dupont, B. Introduction: current concepts in immunity to human cancer and therapeutic antitumor vaccines. Immunol. Rev. 188, 5-8 (2002).

22. Karan, D., Krieg, A. M. \& Lubaroff, D. M. Paradoxical enhancement of CD8 T celldependent anti-tumor protection despite reduced CD8 T cell responses with addition of a TLR9 agonist to a tumor vaccine. Int. J. Cancer 121, 1520-1528 (2007).

23. Saenz-Badillos, J., Amin, S. P. \& Granstein, R. D. RNA as a tumor vaccine: a review of the literature. Exp. Dermatol. 10, 143-154 (2001).

24. Schirrmacher, V. Tumor vaccine design: concepts, mechanisms, and efficacy testing. Int. Arch. Allergy Immunol. 108, 340-344 (1995).

25. Dalgleish, A. G. \& Whelan, M. A. Cancer vaccines as a therapeutic modality: the long trek. Cancer Immunol. Immunother. 55, 1025-1032 (2006).

26. Fong, L. \& Small, E. J. Immunotherapy for prostate cancer. Curr. Oncol. Rep. 9, 226-233 (2007).

27. Minev, B. R., Guo, F., Gueorguieva, I. \& Kaiser, H. E. Vaccines for immunotherapy of breast cancer and prostate cancer: new developments and comparative aspects. In Vivo 16, 405-415 (2002).

28. Simons, J. W. \& Sacks, N. Granulocytemacrophage colony-stimulating factor-transduced allogeneic cancer cellular immunotherapy: the GVAX vaccine for prostate cancer. Urol. Oncol. 24, 419-424 (2006).

29. US National Library of Medicine. ClinicalTrials.gov [online], http://clinicaltrials.gov/ct2/show/ NCT01341652. Randomized phase II trial of a DNA vaccine encoding prostatic acid phosphatase (pTVG-HP) versus GM-CSF adjuvant in patients with non-metastatic prostate cancer (2012).

30. Lonchay, C. et al. Correlation between tumor regression and $\mathrm{T}$ cell responses in melanoma patients vaccinated with a MAGE antigen. Proc Natl Acad. Sci. USA 101, 14631-14638 (2004).

31. Rosenberg, S. A., Yang, J. C. \& Restifo, N. P. Cancer immunotherapy: moving beyond current vaccines. Nat. Med. 10, 909-915 (2004).

32. Sharma, P., Wagner, K., Wolchok, J. D. \& Allison, J. P. Novel cancer immunotherapy agents with survival benefit: recent successes and next steps. Nat. Rev. Cancer 11, 805-812 (2011).

33. Chambers, C. A., Kuhns, M. S., Egen, J. G. \& Allison, J. P. CTLA-4-mediated inhibition in regulation of T cell responses: mechanisms and manipulation in tumor immunotherapy. Annu. Rev. Immunol. 19, 565-594 (2001).

34. May, K. F. Jr, Gulley, J. L., Drake, C. G., Dranoff, G. \& Kantoff, P. W. Prostate cancer immunotherapy. Clin. Cancer Res. 17, 5233-5238 (2011).

35. Small, E. J. et al. A pilot trial of CTLA-4 blockade with human anti-CTLA-4 in patients with hormone- refractory prostate cancer. Clin. Cancer Res. 13 1810-1815 (2007).

36. Chen, H. et al. Anti-CTLA-4 therapy results in higher CD4+ICOShi T cell frequency and IFNgamma levels in both nonmalignant and malignant prostate tissues. Proc. Natl Acad. Sci. USA 106, 2729-2734 (2009).

37. Ribas, A. et al. Phase III, open-label, randomized, comparative study of tremelimumab (CP-675, 206) and chemotherapy [TMZ] or dacarbazine [DTIC] in patients with advanced melanoma. Presented at the ASCO meeting. Abstract LBA9011 (2008).

38. Hodi, F. S. et al. Improved survival with ipilimumab in patients with metastatic melanoma. N. Engl. J. Med. 363, 711-723 (2010).

39. Fong, L. et al. Potentiating endogenous antitumor immunity to prostate cancer through combination immunotherapy with CTLA4 blockade and GM-CSF. Cancer Res. 69, 609-615 (2009).

40. Madan, R. A. et al. Ipilimumab and a poxviral vaccine targeting prostate-specific antigen in metastatic castration-resistant prostate cancer: a phase 1 dose-escalation trial. Lancet Oncol. http://dx.doi.org/10.1016/S14702045(12)70006-2.

41. van den Eertwegh, A. J. et al. Combined immunotherapy with granulocyte-macrophage colony-stimulating factor-transduced allogeneic prostate cancer cells and ipilimumab in patients with metastatic castration-resistant prostate cancer: a phase 1 dose-escalation trial. Lancet Oncol. http://dx.doi.org/10.1016/S14702045(12)70007-4.

42. US National Library of Medicine. ClinicalTrials.gov [online], http://clinicaltrials.gov/ct2/show/ NCT01057810 (2012).

43. US National Library of Medicine. ClinicalTrials.gov [online], http://clinicaltrials.gov/ct2/show/ NCT00861614 (2012)

44. Fizazi, K. et al. Denosumab versus zoledronic acid for treatment of bone metastases in men with castration-resistant prostate cancer: a randomised, double-blind study. Lancet $\mathbf{3 7 7}$, 813-822 (2011).

45. Steinman, R. M. \& Idoyaga, J. Features of the dendritic cell lineage. Immunol. Rev. 234, 5-17 (2010).

46. Ueno, H. et al. Harnessing human dendritic cell subsets for medicine. Immunol. Rev. 234, 199-212 (2010).

47. Thomas-Kaskel, A. K., Waller, C. F., SchultzeSeemann, W. \& Veelken, H. Immunotherapy with dendritic cells for prostate cancer. Int. J. Cancer 121, 467-473 (2007).

48. Waeckerle-Men, Y. et al. Dendritic cell-based multi-epitope immunotherapy of hormonerefractory prostate carcinoma. Cancer Immunol. Immunother. 55, 1524-1533 (2006).

49. Gilboa, E. \& Vieweg, J. Cancer immunotherapy with mRNA-transfected dendritic cells. Immunol. Rev. 199, 251-263 (2004).

50. Heiser, A. et al. Autologous dendritic cells transfected with prostate-specific antigen RNA stimulate CTL responses against metastatic prostate tumors. J. Clin. Invest. 109, 409-417 (2002).

51. Kantoff, P. W. et al. Sipuleucel-T immunotherapy for castration-resistant prostate cancer. N. Engl. J. Med. 363, 411-422 (2010).

52. Centers for Medicare and Medicaid Services [online], https://www.cms.gov/ determinationprocess/downloads/id77TA.pdf. Technology assessment: outcomes of sipuleucel-T therapy (2011).

53. Sheikh, N. A. \& Jones, L. A. CD54 is a surrogate marker of antigen presenting cell activation.
Cancer Immunol. Immunother. 57, 1381-1390 (2008).

54. US National Library of Medicine. ClinicalTrials.gov [online], http://clinicaltrials.gov/ct2/show/ NCT00715078 (2011).

55. US National Library of Medicine. ClinicalTrials.gov [online], http://clinicaltrials.gov/ct2/show/ NCT01431391 (2012).

56. US National Library of Medicine. ClinicalTrials.gov [online], http://clinicaltrials.gov/ct2/show/ NCT01487863 (2012).

57. Paterson, Y., Guirnalda, P. D. \& Wood, L. M. Listeria and Salmonella bacterial vectors of tumorassociated antigens for cancer immunotherapy. Semin. Immunol. 22, 183-189 (2010).

58. Singh, R. \& Paterson, Y. Listeria monocytogenes as a vector for tumor-associated antigens for cancer immunotherapy. Expert Rev. Vaccines 5, 541-552 (2006).

59. Wallecha, A. et al. Multiple effector mechanisms induced by recombinant Listeria monocytogenes anticancer immunotherapeutics. Adv. Appl. Microbiol. 66, 1-27 (2009).

60. Bahjat, K. S. et al. Cytosolic entry controls $\mathrm{CD} 8(+)-\mathrm{T}$-cell potency during bacterial infection. Infect. Immun. 74, 6387-6397 (2006).

61. Chen, Y. et al. Development of a Listeria monocytogenes-based vaccine against hepatocellular carcinoma. Oncogene 31, 2140-2152 (2011).

62. Tangney, M. \& Gahan, C. G. Listeria monocytogenes as a vector for anti-cancer therapies. Curr. Gene Ther. 10, 46-55 (2010).

63. Singh, R. \& Wallecha, A. Cancer immunotherapy using recombinant Listeria monocytogenes: transition from bench to clinic. Human Vaccines 7 , 497-505 (2011).

64. Seavey, M. M. et al. A novel human Her-2/neu chimeric molecule expressed by Listeria monocytogenes can elicit potent HLA-A2 restricted CD8-positive T cell responses and impact the growth and spread of Her-2/neupositive breast tumors. Clin. Cancer Res. 15, 924-932 (2009).

65. Shahabi, V. et al. Development of a Listeria monocytogenes based vaccine against prostate cancer. Cancer Immunol. Immunother. $\mathbf{5 7}$, 1301-1313 (2008).

66. Wallecha, A., Maciag, P. C., Rivera, S., Paterson, Y \& Shahabi, V. Construction and characterization of an attenuated Listeria monocytogenes strain for clinical use in cancer immunotherapy. Clin. Vaccine Immunol. 16, 96-103 (2009).

67. Le, D. T. et al. A live-attenuated Listeria vaccine (ANZ-100) and a live-attenuated Listeria vaccine expressing mesothelin (CRS-207) for advanced cancers: phase 1 studies of safety and immune induction. Clin. Cancer Res. 18, 858-868 (2011).

68. Maciag, P. C., Radulovic, S. \& Rothman, J. The first clinical use of a live-attenuated Listeria monocytogenes vaccine: a Phase I safety study of Lm-LLO-E7 in patients with advanced carcinoma of the cervix. Vaccine 27, 3975-3983 (2009).

69. Kantoff, P. W. et al. Overall survival analysis of a phase II randomized controlled trial of a poxviralbased PSA-targeted immunotherapy in metastatic castration-resistant prostate cancer. J. Clin. Oncol. 28, 1099-1105 (2010).

70. Kaufman, H. L. et al. Phase II randomized study of vaccine treatment of advanced prostate cancer (E7897): a trial of the Eastern Cooperative Oncology Group. J. Clin. Oncol. 22, 2122-2132 (2004).

71. Gulley, J. L. et al. Immunologic and prognostic factors associated with overall survival employing a poxviral-based PSA vaccine in metastatic castrate-resistant prostate cancer. Cancer Immunol. Immunother. 59, 663-674 (2010). 
72. US National Library of Medicine. ClinicalTrials.gov [online], http://www.clinicaltrials.gov/ct2/show/ NCT00450619 (2012)

73. US National Library of Medicine. ClinicalTrials.gov [online], http://www.clinicaltrials.gov/ct2/show/ NCT01322490 (2012).

74. US National Library of Medicine. ClinicalTrials.gov [online], http://clinicaltrials.gov/ct2/show/ NCT00450463 (2012).

75. Babiuk, L. A. \& Tikoo, S. K. Adenoviruses as vectors for delivering vaccines to mucosal surfaces. J. Biotechnol. 83, 105-113 (2000).

76. Connolly, J. B. Conditionally replicating viruses in cancer therapy. Gene Ther. 10, 712-715 (2003).

77. Zhang, W. W. Development and application of adenoviral vectors for gene therapy of cancer. Cancer Gene Ther. 6, 113-138 (1999).

78. Lubaroff, D. M. et al. Decreased cytotoxic T cell activity generated by co-administration of PSA vaccine and $\mathrm{CpG}$ ODN is associated with increased tumor protection in a mouse model of prostate cancer. Vaccine 24, 6155-6162 (2006).

79. Elzey, B. D., Siemens, D. R., Ratliff, T. L. \& Lubaroff, D. M. Immunization with type 5 adenovirus recombinant for a tumor antigen in combination with recombinant canarypox virus (ALVAC) cytokine gene delivery induces destruction of established prostate tumors. Int. J. Cancer 94, 842-849 (2001).

80. Lubaroff, D. Vaccine immunotherapy for prostate cancer [online], http://www.dtic.mil/cgi-bin/GetT RDoc? Location $=\mathrm{U} 2 \&$ doc $=$ GetTRDoc pdf\&AD=ADA462829 (2006).

81. Tatsis, N. et al. Adenoviral vectors persist in vivo and maintain activated CD8+ T cells: implications for their use as vaccines. Blood 110, 1916-1923 (2007)

82. Ilan, Y. et al. Oral tolerization to adenoviral proteins permits repeated adenovirus-mediated gene therapy in rats with pre-existing immunity to adenoviruses. Hepatology 27, 1368-1376 (1998)

83. Anderson, R. D., Haskell, R. E., Xia, H., Roessler, B. J. \& Davidson, B. L. A simple method for the rapid generation of recombinant adenovirus vectors. Gene Ther. 7, 1034-1038 (2000).

84. Lusky, M. Good manufacturing practice production of adenoviral vectors for clinical trials. Hum. Gene Ther. 16, 281-291 (2005).

85. Tatsis, N. \& Ertl, H. C. Adenoviruses as vaccine vectors. Mol. Ther. 10, 616-629 (2004).

86. Li, W. et al. Human colorectal cancer (CRC) antigen C017-11A/GA733 encoded by adenovirus inhibits growth of established CRC cells in mice. J. Immunol. 159, 763-769 (1997).

87. Liu, D. W. et al. Induction of CD8 T cells by vaccination with recombinant adenovirus expressing human papillomavirus type 16 E5 gene reduces tumor growth. J. Virol. 74 , 9083-9089 (2000).

88. Rosenberg, S. A. et al. Immunizing patients with metastatic melanoma using recombinant adenoviruses encoding MART-1 or gp100 melanoma antigens. J. Natl Cancer Inst. 90 1894-1900 (1998).

89. Kim, S., Lee, J. B., Lee, G. K. \& Chang, J. Vaccination with recombinant adenoviruses and dendritic cells expressing prostate-specific antigens is effective in eliciting CTL and suppresses tumor growth in the experimental prostate cancer. Prostate 69, 938-948 (2009).

90. Stratford-Perricaudet, L. D., Levrero, M. Chasse, J. F., Perricaudet, M. \& Briand, P. Evaluation of the transfer and expression in mice of an enzyme-encoding gene using a human adenovirus vector. Hum. Gene Ther. 1, 241-256 (1990).
91. Muona, K., Makinen, K., Hedman, M., Manninen, H. \& Yla-Herttuala, S. 10-year safety follow-up in patients with local VEGF gene transfer to ischemic lower limb. Gene Ther. 19, 392-395 (2011).

92. Sheets, R. L. et al. Biodistribution and toxicological safety of adenovirus type 5 and type 35 vectored vaccines against human immunodeficiency virus-1 (HIV-1), Ebola, or Marburg are similar despite differing adenovirus serotype vector, manufacturer's construct, or gene inserts. J. Immunotoxicol. 5, 315-335 (2008).

93. Casimiro, D. R. et al. Comparative immunogenicity in rhesus monkeys of DNA plasmid, recombinant vaccinia virus, and replication-defective adenovirus vectors expressing a human immunodeficiency virus type 1 gag gene. J. Virol. 77, 6305-6313 (2003).

94. Sumida, S. M. et al. Neutralizing antibodies and CD8+ T lymphocytes both contribute to immunity to adenovirus serotype 5 vaccine vectors. J. Virol. 78, 2666-2673 (2004).

95. Siemens, D. R. et al. Cutting edge: restoration of the ability to generate CTL in mice immune to adenovirus by delivery of virus in a collagen-based matrix. J. Immunol. 166, 731-735 (2001).

96. Lubaroff, D. M. et al. Phase I clinical trial of an adenovirus/prostate-specific antigen vaccine for prostate cancer: safety and immunologic results. Clin. Cancer Res. 15, 7375-7380 (2009).

97. US National Library of Medicine. ClinicalTrials.gov [online], http://clinicaltrials.gov/ct2/show/ NCT00583752 (2012)

98. US National Library of Medicine. ClinicalTrials.gov [online], http://clinicaltrials.gov/ct2/show/ NCT00583024 (2012)

99. Lubaroff, D. et al. An ongoing phase II trial of an adenovirus/PSA vaccine for prostate cancer. Presented at the 2012 American Association for Cancer Research Annual Meeting.

100. Robson, N. C., Hoves, S., Maraskovsky, E. \& Schnurr, M. Presentation of tumour antigens by dendritic cells and challenges faced. Curr. Opin. Immunol. 22, 137-144 (2010).

101. Engell-Noerregaard, L., Hansen, T. H., Andersen, M. H., Thor Straten, P. \& Svane, I. M. Review of clinical studies on dendritic cell-based vaccination of patients with malignant melanoma: assessment of correlation between clinical response and vaccine parameters. Cancer Immunol. Immunother. 58, 1-14 (2009).

102. Adema, G. J., de Vries, I. J., Punt, C. J. \& Figdor, C. G. Migration of dendritic cell based cancer vaccines: in vivo veritas? Curr. Opin. Immunol. 17, 170-174 (2005).

103. Kim, Y. S. et al. CD40-targeted recombinant adenovirus significantly enhances the efficacy of antitumor vaccines based on dendritic cells and B cells. Hum. Gene Ther. 21, 1697-1706 (2010).

104. Hangalapura, B. N. et al. Potent antitumor immunity generated by a CD40-targeted adenoviral vaccine. Cancer Res. 71, 5827-5837 (2011).

105. Brossart, P., Goldrath, A. W., Butz, E. A., Martin, S. \& Bevan, M. J. Virus-mediated delivery of antigenic epitopes into dendritic cells as a means to induce CTL. J. Immunol. 158, 3270-3276 (1997).

106. Karan, D. et al. Dual antigen target-based immunotherapy for prostate cancer eliminates the growth of established tumors in mice. Immunotherapy 3, 735-746 (2011).

107. Medin, J. A. et al. Efficient transfer of PSA and PSMA cDNAs into DCs generates antibody and T cell antitumor responses in vivo. Cancer Gene Ther. 12, 540-551 (2005).

108. Melief, C. J. \& van der Burg, S. H. Immunotherapy of established (pre)malignant disease by synthetic long peptide vaccines. Nat. Rev. Cancer 8, 351-360 (2008)

109. Kottke, T. et al. Broad antigenic coverage induced by vaccination with virus-based cDNA libraries cures established tumors. Nat. Med. 17, 854-859 (2011)

110. Simons, J. W. et al. Phase I/II trial of an allogeneic cellular immunotherapy in hormonenaive prostate cancer. Clin. Cancer Res. 12, 3394-3401 (2006).

111. Small, E. et al. A phase III trial of GVAX immunotherapy for prostate cancer in combination with docetaxel versus docetaxel plus prednisone in symptomatic, castrationresistant prostate cancer (CRPC). Presented at Genitourinary Cancers Symposium. Abstract 7 (2009).

112. Harzstark, A. L. \& Small, E. J. Immunotherapeutics in development for prostate cancer. Oncologist 14, 391-398 (2009).

113. Risk, M. \& Corman, J. M. The role of immunotherapy in prostate cancer: an overview of current approaches in development. Rev. Urol. 11, 16-27 (2009).

114. Dannull, J. et al. Prostate stem cell antigen is a promising candidate for immunotherapy of advanced prostate cancer. Cancer Res. 60, 5522-5528 (2000).

115. Gu, Z. et al. Prostate stem cell antigen (PSCA) expression increases with high gleason score advanced stage and bone metastasis in prostate cancer. Oncogene 19, 1288-1296 (2000).

116. Raff, A. B., Gray, A. \& Kast, W. M. Prostate stem cell antigen: a prospective therapeutic and diagnostic target. Cancer Lett. 277, 126-132 (2009).

117. Reiter, R. E. et al. Prostate stem cell antigen: a cell surface marker overexpressed in prostate cancer. Proc. Natl Acad. Sci. USA 95, 1735-1740 (1998)

118. US National Library of Medicine. ClinicalTrials. gov [online], http://clinicaltrials.gov/ct2/show/ NCT01560923 (2012).

119. US National Library of Medicine. ClinicalTrials. gov [online], http://clinicaltrials.gov/ct2/show/ NCT01145508 (2011)

120. Topalian, S. L., Weiner, G. J. \& Pardoll, D. M. Cancer immunotherapy comes of age. J. Clin Oncol. 29, 4828-4836 (2011).

121. Mellman, I., Coukos, G. \& Dranoff, G. Cance immunotherapy comes of age. Nature 480 , 480-489 (2011)

122. Disis, M. L. Immunologic biomarkers as correlates of clinical response to cance immunotherapy. Cancer Immunol. Immunother. 60, 433-442 (2011)

123. Whiteside, T. L., Gulley, J. L., Clay, T. M. \& Tsang, K. Y. Immunologic monitoring of cellular immune responses in cancer vaccine therapy. J. Biomed. Biotechnol. doi:10.1155/2011/370374.

124. Gulley, J. L. \& Drake, C. G. Immunotherapy for prostate cancer: recent advances, lessons learned, and areas for further research. Clin. Cancer Res. 17, 3884-3891 (2011).

Acknowledgements

Funding for this research was supported by the Hearst Foundation and the William-Valk Chairman's Fund.

Author contributions

D. Karan researched and drafted the manuscript and discussed the contents with colleagues.

J. Holzbeierlein and P. Van Veldhuizen also contributed towards writing the article. All authors reviewed and edited the manuscript prior to submission. 